\title{
A Theoretical Framework to Foster Digital Literacy: The Case of Digital Learning Resources
}

\author{
Said Hadjerrouit \\ University of Agder, Serviceboks 422, \\ 4604 Kristiansand, Norway \\ Said.Hadjerrouit@uia.no
}

\begin{abstract}
The New National Curriculum in Norway from 2006 recommends that teachers and students acquire a high degree of digital literacy in school education. One way to foster digital literacy is to train teachers to design and critically evaluate digital learning resources (DLRs). However, little research has been done as to which design principles and evaluation criteria are suitable for DLRs. This work proposes a theoretical framework, along with a case study, for designing and evaluating DLRs. The article draws on research in learning theory, pedagogical usability criteria, and context of use. The paper reports on the implications of the framework for the design and evaluation of DLRs.
\end{abstract}

Keywords: Digital literacy, digital learning resources, DLR, learning theory, pedagogical usability.

\section{Introduction}

Technical skills are obviously important elements of the digital literacy concept, but a closer look at the concept shows that it is more than the ability to use computers $[1,2,3,4]$. For teachers, digital literacy must include a suitable combination of pedagogical, cognitive, and evaluation skills such as knowledge of how DLRs can support pedagogical goals and knowledge of how the subject matter is transformed by the application of DLRs. Likewise, the ability to use DLRs is important to students, but not sufficient for acquiring a high-level of digital literacy skills. They also need cognitive proficiencies that help them to critically evaluate the information provided by DLRs. Clearly, digital literacy must go beyond the simple use of DLRs, and include pedagogical competencies and cognitive capabilities. However, little research has been done as to which framework is suitable to address issues that are pertinent to these dimensions under the concept of digital literacy.

The research goal of this work is to use the literature, along with a case study, to develop a framework of critical elements in DLRs design and evaluation. First, a literature review is undertaken to report on the state of research in the field of DLRs. Second, a theoretical framework is developed based on the literature review. Then, preliminary experiences with the framework are presented. Finally, some remarks on the value of the framework and elements of a future research conclude the article. 


\section{The Concept of Digital Learning Resources}

A closer look at the concept of "DLR" shows that it is similar to "Web-based learning tools" found in [5], which refers to [6] to define the term as "interactive Web-based tools that support learning by enhancing, amplifying, and guiding the cognitive processes of learners". In addition, the concept of "DLRs" includes the main features of the term "Web-based learning application" defined by Liu \& Johnson [7]. Accordingly, DLRs can be defined as a technology with four dimensions: (a) It is delivered through the Web; (b) It teaches content that meets specific learning objectives aligned with the curriculum; (c) It is designed on the basis of a learning theory; (d) It contains reusable elements.

From the content point of view, DLRs are computer-based implementations of a specific topic that is aligned with a given curriculum. DLRs include study material, task-based activities and exercises, and eventually assessment procedures. DLRs can also be created to support different topics of a given subject, as well as instructional material in a number of subject areas at all levels in school education.

From a pedagogical point of view, the design of DLRs is embedded within a pedagogical strategy associated with a learning theory, such as cognitivism, constructivism, and collaborative learning or a combination of them [9]. The underlying pedagogical strategy of DLRs can affect teaching and learning processes.

Finally, DLRs need to be reusable in order to suit the students' needs. Reusability is useful for teaching subjects in different classroom contexts. Hence, reusability assumes that elements of DLRs can be found to fit into a similar teaching unit [10]. It also assumes that a given teaching unit will find DLRs or elements of them from many Web-based sources or throughout a learning resource database repository.

\section{Literature Review}

Despite the importance of digital literacy in the curriculum, schools adapt slowly to technological and pedagogical changes. According to the research literature, schools are still confronted with a number of problems associated with the realization of digital literacy within the field of DLRs. Basically, the main research issues are the following.

First, DLRs are still the domain of technical and software experts rather than teachers, educators, and learners [11]. As a result, most DLRs basically emphasize technical usability as defined by Nielsen $[12,13]$. Technical usability is important to minimize the cognitive load, and helps to free more resources for the learning process itself. It enables learners to easily focus on learning materials without having to make an effort to figure how to access them. However, although technical usability is a selfevident requirement for DLRs, it is not necessarily conductive for deep learning.

Second, to support learning, research in education $[14,15,16,17]$ suggests that developers need to design DLRs with a new dimension of usability. This dimension is called "pedagogical usability" and it is associated with aspects that are fundamental to learning. However, little attention has been paid to pedagogical usability of DLRs, which is a critical factor to the success of this technology in classroom. From the viewpoint of pedagogical usability, current DLRs lack a number of features that would make them more flexible, interactive, motivating, and collaborative. Research 
literature reveals that DLRs with advanced features are difficult to design, and therefore current systems are still limited in their pedagogical usability. Martinidale et al [9] stated that it is substantially more difficult to create DLRs that accommodate the demands of constructivist learning. Likewise, Liu \& Johnson [7] found a lack of fit between existing DLRs and what teachers and learners need, as well as a lack of connection between DLR design and educational standards.

Third, the pedagogical value of DLRs lies in helping learners discover and explore things for themselves through interactive, flexible, differentiated, and motivating activities. Unfortunately, most DLRs provide little support to achieve a high level of activity [7,9]. This because most DLRs are developed without a previous analysis of learners' needs. Clearly, a learner-centered approach to DLRs requires a change from teacher-centered design to a model of design that emphasizes the learners' needs [18]. Indeed, a learner-centered approach offers more scope for realizing possible learning benefits than traditional instructional paradigms. However, Maddux [19] indicated that such a change demands a massive shift in values related to school culture, teaching, and learning, as well as an intensive commitment to differentiated learning.

Fourth, the integration of DLRs into classroom is strongly connected to teachers' epistemological orientations, personal theories and perceptions about teaching and learning processes. According to Jimoyiannis \& Komis [20], a true integration requires that teachers consider technology, content, and pedagogy not in isolation, but rather in the complex relationships in the system defined by three key components: (a) Knowledge of the pedagogy that is applicable to the specific content; (b) Knowledge of how technology can support pedagogical goals; and (c) Knowledge of how the subject matter is transformed by the application of the technology. Clearly, teachers' technical knowledge alone is not sufficient to achieve learning outcomes using DLRs. Equally important is how DLRs can support pedagogical goals set by the teacher and how the content is transformed through the technology [18]. Clearly, teacher's epistemological orientations should not be underemphasized when it comes to design, implement, and evaluate pedagogical situations in classroom around DLRs.

Fifth, in contrast to higher education, little research has been done evaluating students' and teachers' perceptions of DLRs in school education, modalities of teaching and learning using these resources [5]. In addition, exhaustive instruments (e.g., [21]) may not be suitable to evaluate DLRs, which tend to be smaller and more numerous [16]. Furthermore, a number of evaluation frameworks that can be used to evaluate user interfaces have been proposed [13]. But, these frameworks are intended for software environments rather than DLRs in which learner-centered user interface systems should be developed to support users' activities. Another problem with evaluating DLRs is that relatively few are currently being used in schools. ITU Monitor [22] reports that the selection of DLRs is limited, and that designing, developing, and implementing DLRs, which provide added value in learning and teaching, is very demanding and time consuming. Nevertheless, some schools have made considerable progress in the use of DLRs, but many of them still have much to do to develop and use subject-specific digital DLRs.

Summarizing, the literature review reveals a lack of design and evaluation regarding DLRs in educational settings. This work sets out to address this by developing a theoretical framework for the identification of educational factors that are important to the design and evaluation of DLRs. 


\section{A Theoretical Framework for Design and Evaluation of DLRs}

The process of designing and evaluating DLRs in school education is a many-sided field of study with a number of theoretical and practical issues. Two fields are of interest for developing a coherent theoretical framework for designing and evaluating DLRs: Pedagogical usability issues and the context of use and evaluation.

\subsection{Pedagogical Usability Criteria}

The concept of usability has been defined by a number of researchers, but a complete definition is difficult to achieve, outside the domain it is to be considered [23,24]. Nielsen [12,13], as one of the foremost usability researchers, proposed a definition that focuses on technical usability, which involves criteria such as the need for consistency, learner satisfaction, minimal user actions and minimal memory load, simplicity and reduction of complexity. However, the impact of Nielsen's definition is limited when it comes to DLRs, because it largely ignores the underlying processes of human learning. Clearly, DLRs are considered beneficial for the learners if they contribute to the learning process and not if they simply support technical tasks. Hence, the usability of DLRs must be re-defined and related to its learning utility [25]. In order to reformulate the concept, it must be directly related to learning theories [26]. The literature on learning theories points to the fundamental differences between them [27], but in school education, a mix of learning theories is being used. Hence, teachers must allow circumstances surrounding the learning situation to help them decide which approach to learning is the most appropriate [28]. In the case of DLRs, designers need to consider that this technology suggests more a learner-centered approach than teacher-centered methods [18] in order to realize learning benefits, since the goal is to support the learners' knowledge construction process.

Accordingly, Nokelainen [17] expanded Nielsen's definition to include pedagogical usability issues. Nokelainen defined a set of criteria that can be applied to DLRs: learner control, learning activity, collaborative learning, goal orientation, applicability, added value, motivation, previous knowledge, flexibility, and feedback. These criteria can be adapted to DLRs with slight modifications, because DLRs cannot be judged in exactly the same terms as digital material that is used in other contexts. Hence, the key criteria that influence the pedagogical usability of DLRs are:

Understandability. DLRs should provide a well-structured description of the subject information using a clear and understandable language.

Added value. The added value of DLRs means that they offer more learning potentialities than traditional resources, such as textbooks, in terms of the potential capabilities of DLRs such as interactivity, differentiation, etc.

Goal-orientation. This criterion is related to the learning utility of DLRs and meaningfulness in terms of the learning goals set by the teacher and the curriculum.

Time. DLRs must allow the student to learn the subject matter within an acceptable period of time. It should preferably take less time to learn the subject information with the DLR than with textbooks.

Interactivity. DLRs should provide support for interactivity through a user-friendly accessibility of the subject information and task-based activities. Interactivity is important because it allows students to be actively involved in problem solving. 
Multiple representation of information. DLRs should provide multiple representation of information using various multimedia elements. Task-based activities can contain links to multimedia applications such as games and simulations. Students should feel that their learning is enhanced through the use of multimedia.

Motivation. The material provided by DLRs should contain intrinsically motivating tasks. It should show important aspects of the subject matter that may affect the students' learning. Motivation is goal oriented and facilitates students' higher levels of engagement with the study material.

Differentiation. This criterion implies that DLRs are adapted to the students' characteristics. Differentiation involves fitting the subject information to the students' needs, taking into account their abilities, gender, language, needs, prior knowledge, and computer skills.

Flexibility. Flexibility means that DLRs provide different levels of difficulty and contain diverse assignments and tasks that are tailored to all students, so that their individual differences are taken into account.

Autonomy. This criterion means that students are able to work on their own using DLRs, and acquire knowledge without being completely dependent on the teacher. The knowledge provided by DLRs should be potentially powerful to enable the student to become less dependent on the teacher.

Collaboration. This criterion means that students can work together to reach a common goal, giving them a sense of how good solutions can be performed in collaboration. The criterion is important because learning is considered as an inherently social activity as good solutions are developed not in isolation; instead they involve collaboration with other students.

Variation. Students are able to use different learning resources in combination with DLRs. Variation is important to learning, because of different students' learning styles and preferences, or because some students favor some particular methods of interacting with others, while others prefer other approaches.

\subsection{Context of Use and Evaluation of DLRs}

The second key element of the framework is the context of use and evaluation of DLRs. The most appropriate approach to carefully examine the context of DLRs is to look at the influencing factors connected to them. The context is the totality of relationships between the students and surrounding elements within a pedagogical situation. This concept of context is similar to Brousseau's term of "milieu" [29], where learning is described as emerging from exchanges between the students and a "milieu" organized with teaching intentions. Accordingly, milieu is everything in the situation the learners can act on. It can be divided into two categories: Material and non material milieu. The material milieu includes a number of elements:

- The subject matter, its content, topics and subtopics, task-based activities, assignments, exercises, and problem statements taught in the classroom.

- The curriculum and its objectives, main subject areas, basic skills, including computer skills, competence aims in the subject, and subject assessment.

- Infrastructure (technical and non-technical), software tools available, number and place of computers, student/PC-ratio in the classroom.

- Study material such as textbooks, electronic and written material. 
The non material milieu consists of the stakeholders involved in school education:

- Fellow learners in the classroom and their characteristics, such as age, knowledge level, gender, interests, needs, motivations, and computer skills.

- School teachers and their profile and pedagogies, information and communication technology (ICT) qualifications and attitudes towards ICT.

- School leaders, decision makers and actors supporting ICT integration.

- Trainee teachers as designers and developers of DLRs.

The mileu forms the very basis for the process underlying the design, use, and evaluation of DLRs. The milieu may change for the learner when introducing a new element - the DLR, because the learning and teaching processes are then mediated by DLRs. According to Brousseau [29], learning occurs by means of interaction between the learner and the "milieu". Learning happens through adaptation of the learner to the "milieu". This view is consistent with Piaget's work [30] that considers learning as an adaptation process to a milieu. The DLR can be considered as an element of the "milieu", and as such, it becomes a source of learning, by means of interaction with the learner.

The nature of the interaction between learners and DLRs is depending on the instructional approach and epistemological orientation adopted by the teacher. DLRs, by their very nature, require a change from teacher-centered methods to learnercentered approaches in order to achieve learning benefits [18].

\section{Preliminary Experiences with the Framework}

A preliminary case study within teacher education was carried out to investigate the value of the theoretical framework. This was done in collaboration with three classes from a middle school, where trainee teachers designed and evaluated DLRs. The unit of study was the learning of school subjects using the DLRs. Data collection methods consisted of student and teacher survey questionnaires. The aim was to measure the students' and teachers' perceptions of DLRs. Data from the case study came from three cohorts of participants: Sixty-five students between 14-16 years from three different classes, three school teachers, and three teams of trainee teachers as developers of DLRs. From the case study the following implications can be drawn for two major organizing themes:

- Pedagogical usability of DLR design

- Digital literacy and integration of DLRs into classroom

\subsection{Pedagogical Usability and DLR Design}

Pedagogical usability defines the capability of the DLRs to support the learning of the subject matter. Looking at the different dimensions of pedagogical usability some implications can be drawn.

First, learning activities are important from the point of view of the constructivist learning theory. Both students and teachers pointed out that the degree of learning activities was satisfying, but the tasks provided by the DLRs, were not sufficient to 
really engage the students in constructivist learning as they do not cover the entire knowledge level of a number of students who particularly demonstrated strong motivation and interests in the topics. Advanced learning activities for those students can be achieved with increased integration of motivating and multimedia elements.

Second, teachers were not quite satisfied with the degree of collaboration between students, because they felt that the students did not work together to reach a common goal. The idea of collaboration fits well with the socially situated learning theory, which assumes that learning occurs as learners improve and reflect on their understanding of the subject matter through discussion with fellow learners [31]. Accordingly, DLRs should contribute to increase the learning by collaboration in a higher degree, and allow the students to engage in levels of activity that could not be managed alone, without collaboration with fellow students. Of course, DLRs in themselves cannot contribute to collaboration, unless teachers perceive the value of collaborative learning.

Third, differentiation is an important criterion for any DLR. It includes different ways of interacting with the DLR while giving special consideration to differentiation between students according to their ability, the different ways students learn, and the different speeds at which students learn. Since it cannot be expected to develop resources with a high degree of differentiation within a limited period of time, it implies that the students' perceptions of differentiation cannot be generalized even if most students pointed out that they were satisfied with the level of differentiation and flexibility of the learning material. Nevertheless, designers of DLRs should take into account the students' preferred learning styles and knowledge levels.

Fourth, the criterion of motivation measures the degree of engagement of the students with the subject matter when using the DLR. Accordingly, DLRs cannot be considered as highly motivating if they are not adapted to the students' age, development, and interests. Student satisfaction with a DLR is, of course, a subjective phenomenon, but one that is important in keeping students engaged with the subject matter. According to Nokelainen [17], motivation can be achieved in many ways, for example through self-regulation, performance or learning goals, as well as extrinsic or intrinsic goal orientation, such as meaningful and interesting study material provided by the DLRs.

Fifth, the criterion of autonomy measures the degree of students' independence from the teacher when using DLRs. This criterion also measures the degree of independence from textbooks and other learning resources. Hence, autonomy and independence can reveal the quality of information provided by the DLR. However, DLRs can contribute to independent learning only if the added value of this technology is taken into consideration and well-perceived by teachers.

Finally, from the viewpoint of pedagogical usability, the DLRs lack a number of features that would make them more flexible, interactive, motivating, and collaborative. Even if the research literature [7,9] reveals that DLRs with advanced features are difficult to design, and therefore current DLRs are still limited in their pedagogical usability, one may ask whether the teachers really perceived the potentialities and added value of DLRs. This issue has been addressed by the teachers from different points of view. As a result, teachers still perceive textbooks as important as DLRs in terms of learning outcomes. 


\subsection{Digital Literacy and Integration of DLRs into Classroom}

There have been ongoing efforts for many years to bring ICT into classrooms and to integrate digital literacy into the curriculum. Despite these efforts, there is a little success achieved so far [32]. It is obvious that ICT integration is a complex and demanding issue both for schools and teachers [33,20,34]. Besides cultural barriers that are caused by the nature of the educational culture and the culture at large as well, which are very difficult to change, there exist pedagogical barriers, as this work clearly demonstrated. Given this background, there are a number of implications that result from the case study for the integration of DLRs into classroom.

First, the curriculum requires that digital literacy be integrated in all subjects, otherwise teachers and students would not be able to fulfil the requirements given by the curriculum. The case study reveals that teachers clearly see the importance of DLRs, but they still consider them as supplementary aids in addition to textbooks. This means that DLRs can be used in combination with textbooks inside and outside the classroom, but they should not drastically affect teachers' ways of instruction, or change their pedagogy. As a result, teachers still perceive DLRs as neutral elements, without added value, that can be used occasionally, in addition to textbooks. Digital literacy however requires more commitment to the potentialities of DLRs.

Second, it thus is expected that DLRs introduce an added value to the learning process in comparison to printed material such as textbooks and material produced by the teacher. The added value of DLRs is in the form of the potentialities this technology offers, for example interactivity, differentiation, and collaboration, in contrast to textbooks that are more rigid and static in their use. However, DLRs can only be useful if the tasks are adapted to the capabilities of the technology. Tasks should not be a direct translation of study materials found in textbooks. They must be interactive and flexible according to students' individual differences and provide the students with immediate feedback that stimulates the learning motivation.

Third, teachers must be aware of the fact that ICT is not a value-neutral technology [37]. On the contrary, ICT is embedded within pedagogical values that potentially affect teaching and learning processes. It follows that IT can have an effect on how teachers use technology and what students learn from it. Similarly, DLRs cannot be considered as a neutral technology that can be used in classroom without radical changes of the content and ways of teaching and learning. Consequently, the introduction of DLRs can create new pedagogical situations that affect both the students' learning and the teaching content. However, teachers are not always aware of the nonneutrality of DLRs. If DLRs can create new pedagogical situations, then textbooks can no longer play the same role as before. Clearly, the added value of DLRs cannot be compensated by any textbooks or printed material.

Fourth, there is evidence in the research literature that the integration of ICT is associated with a shift from instructivist or teacher-centred to constructivist or learnercentred methods and teaching philosophies [18]. The case study seems to confirm, to a certain degree, that constructivist learning is important, since teachers reported that interactive learning activities and collaboration should be given more consideration. A shift from instructivism to constructivism requires that the teachers' role changes from transmitter of knowledge to facilitator of learning. 
Fifth, another crucial factor that may promote or hinder the integration of DLRs is time. Indeed, research reveals that IT can provide positive learning opportunities, but it takes time [35,36]. Accordingly, the overall impression of researchers is that teachers' success in integrating ICT is highly dependent upon the availability of time to think deeply about pedagogical changes. Time plays also an important role for the students in terms of learning effect compared with printed material. However, the fact that DLRs can be used at any time and place does not necessarily mean that it takes less time to learn the subject matter compared to textbooks.

Finally, according to Nokelainen [17], the integration of DLRs into classrooms must be goal-oriented. This means that the objectives of using DLRs must be clear to the students. It seems that a successful integration can be achieved only if the students' goals, curriculum considerations, learning and teaching material, and the teachers' goals are closely aligned. Goals can differ according to the learning situation. The goal may be the learning of basic knowledge with clearly specified topics. The goal may also be the involvement of students in interactive activities. In addition, the goal may be collaboratively discussing important issues of the subject matter, or evaluating the students' knowledge through graded assessment procedures.

\section{Conclusions and Future Work}

The main research goal of this work is to use the literature, along a case study, to develop a framework of critical elements in DLR design and evaluation to foster digital literacy. To this end the framework was used to inform a case study, and the findings of the study were mapped back onto the framework in order to identify additional features found in classroom setting. As a result of this mapping the following conclusions can be drawn.

First, the main factor that impacted the design and evaluation of DLRs was the pedagogical usability dimension. While students and teachers globally agreed in their views of pedagogical usability, they differed in their perceptions regarding some key criteria: Interactivity, autonomy/independence, collaboration, and variation. The findings highlighted the importance of constructivist learning, both individually and collaboratively, for the design and evaluation of DLRs, and the added value of this technology compared with traditional learning resources. These findings are compatible both with elements of the conceptual framework and literature review.

Second, the case study revealed the existence of cultural values connected to students' preferred choices and ways of learning. Cultural usability is to a certain degree implicitly connected both to the technical and pedagogical usability of DLRs. Although the catalogue of usability criteria does not explicitly address cultural usability [38], a number of students expressed their preferred cultural values through the comments they made by means of survey questionnaires. The case study thus reveals that DLRs cannot be considered as a value-neutral technology [37]. On the contrary, cultural values are embedded within DLRs,

Future work will focus on the refinement of the theoretical framework and associated usability concepts - both pedagogical and cultural - in order to generate a more complete picture of what constitutes digital literacy in the field of DLRs. It is also important to further investigate the factors that influence the integration of DLRs into classroom and the context of use and evaluation as well. 


\section{References}

1. Bruillard, E.: From the Didactics of Computer Science towards the Didactics of Instrumental Activities with ICT. In: Second Greek Conference on Didactics of Informatics, Volos (2004)

2. Drenoyianni, H.: Designing and Implementing a Project-Based ICT Course in a Teacher Education Setting: Rewards and Pitfalls. Education \& Information Technologies 9(4), 387-404 (2004)

3. Krumsvik, R.: The Digital Challenges of School and Teacher Education in Norway: Some Urgent Questions and the Search for Answers. Education and Information Technologies 3\&4(11), 239-256 (2006)

4. Markauskaite, L.: Exploring the Structure of Trainee Teachers' ICT Literacy: The Main Components of and Relationships between, General Cognitive and Technical Capabilities. Education Tech. Research Dev. 55, 547-572 (2007)

5. Kay, R., Knaak, L., Petrarca, D.: Exploring Teachers Perceptions of Web-Based Learning Tools. Interdisciplinary Journal of E-Learning and Learning Objects 5, 27-50 (2009)

6. Agostinho, S., Bennett, S., Lockyer, L., Harper, B.: Developing a Learning Object Metadata Application Profile Based on LOM Suitable for the Australian Higher Education Market. Australian Journal of Educational Technology 20(2), 191-208 (2004)

7. Liu, L., Johnson, D.L.: Web-Based Resources and Applications. Computer in the Schools 21(3), 131-147 (2005)

8. Norton, P., Hathaway, D.: On Its Way to K-12 Classrooms, Web 2.0 Goes to Graduate School. Computers in Schools 25(3), 163-180 (2008)

9. Martinidale, T., Cates, W.M., Qian, Y.: Analysis of Recognized Web-Based Educational Resources. Computers in the Schools 21(3), 101-117 (2005)

10. Strijker, A., Collis, B.: The Influence of Context on the Future of Learning Objects. In: Hartman, K., Koohang, A. (eds.) Learning Objects: Theory, Praxis, Issues, and Trends, pp. 83-112. Informing Science Press, Santa Rosa (2007)

11. Nam, C.S., Tonya, L., Smith-Jackson, T.L.: Web-Based Learning Environment: A TheoryBased Design Process for Development and Evaluation. Journal of Information Technology Education 6, 23-44 (2007)

12. Nielsen, J.: Designing Web Usability: The Practice of Simplicity. New Riders Publishing, Indianapolis (2000)

13. Nielsen, J.: Usability Engineering. Academic Press, Boston (1993)

14. Kukulska-Hulme, A., Shield, L.: Usability and Pedagogical Design: Are Language Learning Web Sites Special? In: Cantoni, L., McLoughlin, C. (eds.) Proceedings of World Conference on Educational Multimedia, Hypermedia and Telecommunications 2004, Chesapeake, VA, USA, pp. 4235-4242 (2004)

15. Laurillard, D.: Rethinking University Teaching: A Conversational Framework for the Effective Use of Learning Technologies, 2nd edn. Routledge, London (2002)

16. Leacock, T.L., Nesbit, J.C.: A Framework for the Quality of Multimedia Resources. Educational Technology \& Society 10(2), 44-59 (2007)

17. Nokelainen, P.: An Empirical Assessment of Pedagogical Usability Criteria for Digital Learning Material with Elementary School Students. Educational Technology \& Society 9(2), 178-197 (2006)

18. John, P., Sutherland, R.: Teaching and Learning with ICT: New Technology, New Pedagogy? Education, Communication \& Information 4(1), 101-107 (2009)

19. Maddux, C.D.: The Web in K-12 Education. Computers in the Schools 21(3), 149-165 (2005) 
20. Jimoyiannis, A., Komis, V.: Examining Teachers' Beliefs about ICT in Education: Implications of a Teacher Preparation Programme. Teacher Development 11(2), 149-173 (2007)

21. Squires, D., Preece, J.: Predicting Quality in Educational Software: Evaluating for Learning, Usability and the Synergy between Them. Interacting with Computers 11, 467-483 (1999)

22. ITU Monitor: The Digital State of Affairs in Norwegian schools (2009), http: / /www. itu.no/ITU+Monitor.9UFRDSXH.ips

23. Simbulan, M.S.: Learning Objects' User Interface. In: Hartman, K., Koohang, A. (eds.) Learning Objects: Applications, Implications, \& Future Directions, pp. 259-336. Informing Science Press, Santa Rosa (2007)

24. Peterson, D.: Usability Theory, Practice and Evaluation for Learning Objects. In: Hartman, K., Koohang, A. (eds.) Learning Objects: Applications, Implications, \& Future Directions, pp. 337-370. Informing Science Press, Santa Rosa (2007)

25. Sedig, K., Klawe, M., Westrom, M.: Role of Interface Manipulation Style and Scaffolding on Cognition and Concept Learning in Learnware. ACM Transactions on ComputerHuman Interaction 8(1), 34-59 (2001)

26. Elliott, G.J., Jones, E., Barker, P.: A Grounded Approach to Modeling Learnability of Hypermedia Authoring Tools. Interacting with Computers 14, 547-574 (2002)

27. Lin, B., Hsieh, C.: Web-Based Teaching and Learner Control: A Research Review. Computers \& Education 37(3-4), 377-386 (2001)

28. Karagiorgi, Y., Symeou, L.: Translating Constructivism into Instructional Design: Potential and Limitations. Educational Technology \& Society 8(1), 17-27 (2005)

29. Brousseau, G.: Theory of Didactical Situations. Kluwer Academic Publishers, London (1998)

30. Piaget, G.: Genetic Epistemology. Basic Books, New York (1971)

31. Vygotsky, L.S.: Thought and Language. In: Translated by Kozulin, A. (ed.) MIT Press, Cambridge (1986) (Original English translation published 1962)

32. Tondeur, J., Van Keer, H., Van Braak, H., Valcke, M.: ICT Integration in the Classroom: Challenging the Potential of a School Policy. Computers \& Education 51, 212-223 (2008)

33. Guelbahar, Y.: Technology Planning: A Roadmap to Successful Technology Integration in Schools. Computers \& Education 49, 943-956 (2005)

34. Hadjihoma, C., Karagiorgi, Y.: The Use of ICT in Primary Schools within Emerging Communities of Implementation. Computers \& Education 52, 83-91 (2009)

35. Hayes, D.N.A.: ICT and Learning: Lessons from Australian Classrooms. Computers \& Education 49, 385-395 (2007)

36. Teartle, P.: A Theoretical and Instrumental Framework for Implementing Change in ICT in Education. Cambridge Journal of Education 34(3), 331-351 (2004)

37. Gadanidis, G., Schindler, K.: Learning Objects, Type II Applications, and Embedded Pedagogical Models. Computers in the Schools 21(1), 19-32 (2006)

38. Li, H., Sun, X., Zhang, K.: Culture-Centered Design: Cultural Factors in Interface Usability and Usability Tests. In: Eighth ACIS International Conference on Software Engineering, Artificial Intelligence, Networking, and Parallel/Distributed Computing, SNPD 2007, pp. 1084-1088 (2007) 